\title{
Special issue for data intensive eScience
}

\author{
Judy Qiu • Dennis Gannon
}

Published online: 25 August 2012

(C) Springer Science+Business Media, LLC 2012

\section{Background}

Data-intensive science is enabled by the data deluge and has been called the "fourth paradigm" of scientific discovery. New fields are being born, such as drug discovery based on large scale study of correlations in published papers and climate implications from data on the accelerating pace of changes in previously quiescent ice sheets. Astronomy and the search for fundamental particles at the Large Hadron Collider drive mainstream aspects of data-intensive science with many petabytes of data derived from large advanced instruments. Medical imagery and genomics have as much data but from a slew of distributed instruments. It is projected that there will be 24 billion devices on the Internet by 2020. Most of this "Internet of Things" will be small sensors that produce streams of information which will be processed and integrated with other streams and turned into knowledge. The deluge and its impact are pervasive. In synergy with moving to a data-driven world, we are also in the midst of an evolution in the compute landscape of hardware systems. We now live in the world of massive multi-core and GPU processing systems, very large main memory systems, fast networking components, fast solid state drive, and large data centers that consume massive amounts of energy. Computing paradigm is changing and suggesting new programming models, new data structures and more attention to fault tolerance while enabling much easier access to computing. It is clear that many aspects of how we have dealt with data processing have to change in this new world.

J. Qiu (凶)

Indiana University, Bloomington, IN, USA

e-mail: xqiu@cs.indiana.edu

D. Gannon

Microsoft Corporation, Seattle, WA, USA

e-mail: Dennis.Gannon@microsoft.com 
The focus of this special issue is on novel data processing techniques for this new data-driven world.

Relevant contributions have been provided by Atkinson et al. [1], by Bui et al. [2], by Gadelha et al. [3], by Graham et al. [4], by Lu et al. [5], and by Nam et al. [6]. These contributions focus on:

- presenting a collaborative framework for data intensive science applications with three important components: a workflow language (DISPEL), a registry with semantic descriptions and a streaming-process model of enactment platform;

- a distributed storage system with meta data services and optimized for robustness of scientific data archiving;

- a provenance query framework that captures runtime execution details for manytask scientific computing workflow tasks and supports protein science and social network analysis;

- addressing the issue of the heterogeneity of data associated with transient astronomical events and how to manage and analyze it;

- GPU-based sequence alignment tools using a filtering-verification algorithm with a mix of the Bi-directional Burrows-Wheeler Transform (Bi-BTW) search, direct matching, and deployment optimizations;

- two novel cache-aware distributed query-scheduling algorithms by simulating queries for scientific analysis applications;

This data intensive eScience special issue encouraged researchers to submit and present original work related to the latest trends in preservation, movement, access and analysis of massive datasets that require new tools to support all aspects of data-intensive investigation in science. Within this overall scope, this special edition emphasized fundamental issues of extracting useful information from large, diverse, distributed and heterogeneous data sets and validations through real world applications and case studies in astronomy, biology, chemistry, physics, and social science. New insights from interdisciplinary approach are generated from new technologies for sharing data and information, which include mathematical algorithms; modeling, prediction and simulation methodology; performance measurements and analysis; languages and semantic tools; cache, memory, storage, and network architecture; CPU and GPU accelerator architectures and fault-tolerant systems.

\section{Special issue papers}

Atkinson et al. [1] present an architecture for data intensive science applications with three important components: a canonical language (DISPEL), a registry and an enactment platform. The work is motivated by separating concerns and engineering, as many scientific experiments are increasingly interdisciplinary and it is challenging to collaborate among domain experts, data analysis experts and data intensive engineers. The architecture is divided into tool level that accommodates applications, tool sets and working practices; data intensive enactment level (data intensive platform) that maps optimization, deployment and execution; and an interface between them using DISPEL to describe a workflow. The proposed collaborative framework 
can potentially promote knowledge discovery process on large-scale distributed and heterogeneous data in scientific (Astronomy, Biology, Seismology, Environmental Management) and business domains.

Bui et al. [2], describe the design and implementation of ROARS, a robust object archival system for storing scientific data with the write-once-read-many access mode. By using a relational database to help manage structured metadata and building a distributed file system to store binary data, ROARS is able to support both a rich set of query operations on metadata, and robust storage of binary file data with high access performance. The metadata are stored in the file system for purposes of failure recovery and consistency check. High availability and scalability are achieved by replicating file data on multiple storage nodes in different groups, and supporting dynamic data migration. ROARS protects data integrity with checksum, and provides some other useful features such as materialized views and active storage jobs. Performance evaluations show that ROARS can provide high throughput and low latency comparable to HDFS and has significantly higher metadata query performance.

Gadelha et al. [3] present MTCProv, a provenance management system for manytask scientific computing that focuses on workflow-level provenance architecture and the details of a new query language SPQL and its use on details of execution metadata. It supports for capturing, storing and querying events including re-executions for fault-tolerance, redundant submission of tasks and resource consumption of disk I/O, memory usage and processor load. MTCProv simplifies SQL-like queries by abstracting common provenance query patterns into built-in function. It is validated with a protein structure prediction workflow on a 186-node cluster.

Graham et al. [4] describe some of the informatics challenges in astronomy. Over the past 20 years, astronomy has transitioned from a data-poor science to an immensely data-rich one. The data rate is extremely large in this paper (200$2000 \mathrm{~PB} /$ day), which magnifies the already existing challenges in data handling and exploration. The authors claim RDBMS will not function well beyond $100 \mathrm{~TB}$ level, therefore NoSQL class of distributed storage technologies are necessary. SciDB, a column oriented system which treats arrays as first-class, is a better match. To cope with all these, the concept of Virtual Observatory (VO) is developed, which provides the wherewithal to aggregate and analyze disparate data sets based on data discovery, exploration, fusion, and statistical analysis. The VO includes data federation, data storage, workflows, and semantics. The most interesting aspect of this is the need for a (near) real time mining of massive data streams. The authors describe an event infrastructure that can monitor the sky systematically.

Lu et al. [5] propose G-Aligner, a CPU-GPU based sequence alignment solution for computational genomics applications. The algorithm is composed as a pipeline of Filtering (Bi-BTW search), SA conversion and matching subtasks, where each subtask is a pleasingly parallel execution on CPU (for suffix arrays conversion) or GPU (for filtering and matching). Several optimizations include sorting, cache and thread warps usage. The experiments are conducted on 1 billion alignments using NCBI data set. The performance results of G-Aligner show an improvement of, for instance, a factor of 4 compared with SOAP3.

Finally, Nam et al. [6] present two novel cache-aware distributed query-scheduling algorithms by simulating queries for scientific analysis applications. These ap- 
proaches are built on top of the existing Exponential Moving Average (EMA) statistical prediction method, which does a good job in reusing the cache for distributed querying. However EMA is not ideal when the query distribution has hot spots and when the query distribution pattern changes frequently. The two proposed approaches are focused on addressing these shortcomings of the EMA method. The authors propose a new scheduling approach, Balanced Exponential Moving Average (BEMA), optimized for query scheduling with both load balance and cache-hit ratio considerations. The authors present performance evaluation showing that the two new proposed approaches out-perform the EMA approach for the workloads with hotspots and for changing workloads.

Acknowledgements We would like to thank the authors for contributing papers on their research on latest trends in data intensive technologies and applications for this special issue, and thank all the reviewers for providing constructive reviews and in helping to shape this special issue. Finally we would like to thank the editors of Distributed and Parallel Databases for providing us an opportunity to bring this special issue to the research community.

\section{References}

1. Atkinson, M., Liew, C.S., Galea, H., Martin, P., Krause, A., Mouat, A., Corcho, O., Snelling, D.: Dataintensive architecture for scientific knowledge discovery. Special Issue on Data Intensive eScience of Distributed and Parallel Databases

2. Bui, H., Bui, P., Flynn, P., Thain, D.: ROARS: a robust object archival system for data intensive scientific computing. Special Issue on Data Intensive eScience of Distributed and Parallel Databases

3. Gadelha, L., Wilde, M., Mattoso, M., Foster, I.: MTCProv: a practical provenance query framework for many-task scientific computing. Special Issue on Data Intensive eScience of Distributed and Parallel Databases

4. Graham, M.J., Djorgovski, S.G., Mahabal, A., Donalek, C., Drake, A., Longo, G.: Data challenges of time domain astronomy. Special Issue on Data Intensive eScience of Distributed and Parallel Databases

5. Lu, M., Tan, Y., Bai, G., Luo, Q.: High-performance short sequence alignment with GPU acceleration. Special Issue on Data Intensive eScience of Distributed and Parallel Databases

6. Nam, B., Hwang, D., Kim, J., Shin, M.: High-throughput query scheduling with spatial clustering based on distributed exponential moving average. Special Issue on Data Intensive eScience of Distributed and Parallel Databases 